Cómo citar este artículo / How to cite this article: Baena del Alcázar, L. (2020). Sobre un torso masculino en el Museo de Santa Cruz (Toledo). Lucentum, XXXIX, 169-176. https://doi.org/10.14198/LVCENTVM2020.39.09

\title{
SOBRE UN TORSO MASCULINO EN EL MUSEO DE SANTA CRUZ (TOLEDO)
}

\author{
STUDY OF A MALE TORSO IN THE MUSEUM OF SANTA CRUZ (TOLEDO)
}

LUIS BAENA DEL ALCÁZAR

Universidad de Málaga

lbaena@uma.es

https://orcid.org/0000-0002-2966-2468

Recepción: 21-11-2019

Aceptación: 03-05-2020

\section{Resumen}

Estudio de una escultura masculina conservada en los fondos del Museo de Santa Cruz de Toledo que, por su estructura corporal, recuerda creaciones de Policleto, pero cuya tipología se encuadra en esculturas de Praxiteles o de su escuela, las cuales tendrán una gran difusión en época helenística y romana. La identificación de la escultura apunta a la iconografía de Apolo, la cual serviría en alguna villa de la región, muy posiblemente, como elemento decorativo.

Palabras clave. Escultura romana; Toledo; Museo de Santa Cruz.

\begin{abstract}
Study of a male sculpture stored in the Museum of Santa Cruz in Toledo. Its body structure reminds of Polykleito's creations, but the typology fits with the sculptures of Praxiteles or his school, which were widely spread in the Hellenistic and Roman periods. The identification of the sculpture points to the iconography of Apollo, and would probably be a decorative element in some villa in this region.
\end{abstract}

Key words. Roman sculpture; Toledo; Santa Cruz Museum. 
La escultura que se presenta en esta publicación es una pieza conocida de antiguo, pero que ha pasado inadvertida por los investigadores, muy posiblemente por conservarse durante muchos años en los fondos del Museo de Santa Cruz de Toledo ${ }^{1}$. La primera noticia de la escultura se debe a San Román (1941: 153), en una escueta referencia que citamos: «Torso de pequeña estatua romana de figura varonil desnuda, en mármol blanco, de 0,50 $\mathrm{m}$ de altura». De procedencia y contexto arqueológico desconocido, pero muy posiblemente de la provincia de Toledo, ingresó en el Museo depositado por el Servicio de Defensa del Patrimonio Artístico Nacional. Fue publicado por Aragoneses (1957: 106-108; 1958: 69, $\mathrm{n}^{\circ}$ 4) y puesto en valor al mundo científico por Baena del Alcázar (2016: 79-80).

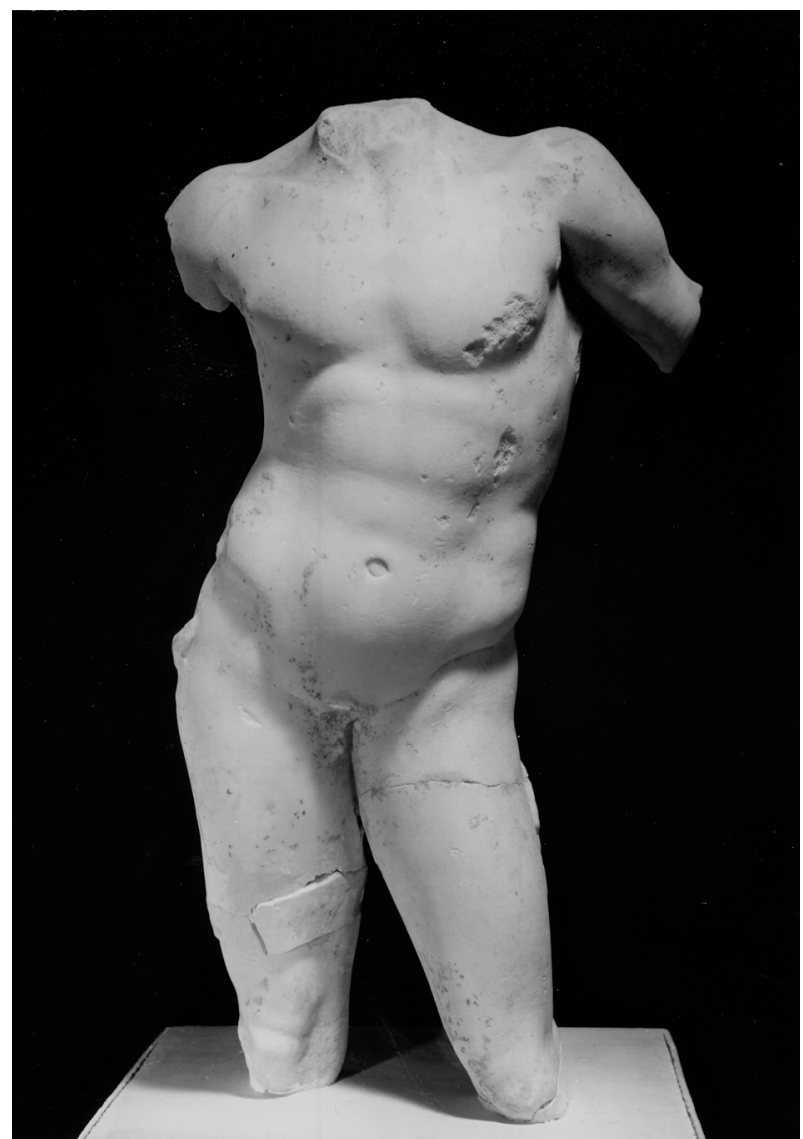

Figura 1: Torso de Toledo. Visión frontal. Foto: Cortesía Prof. J. M. Noguera Celdrán

1. Quedo muy reconocido a D. Jaime Gallardo Alamillo, perteneciente al Departamento de Conservación y Difusión del Museo de Santa Cruz, por su gentileza al proporcionarme datos de la pieza y las primeras fotografías para su estudio. Agradecemos igualmente al Prof. José Miguel Noguera Celdrán por habernos cedido las cuatro primeras fotografías que ilustran este trabajo, sin olvidar a los evaluadores que nos han aportado interesantes sugerencias para la mejora de esta investigación.

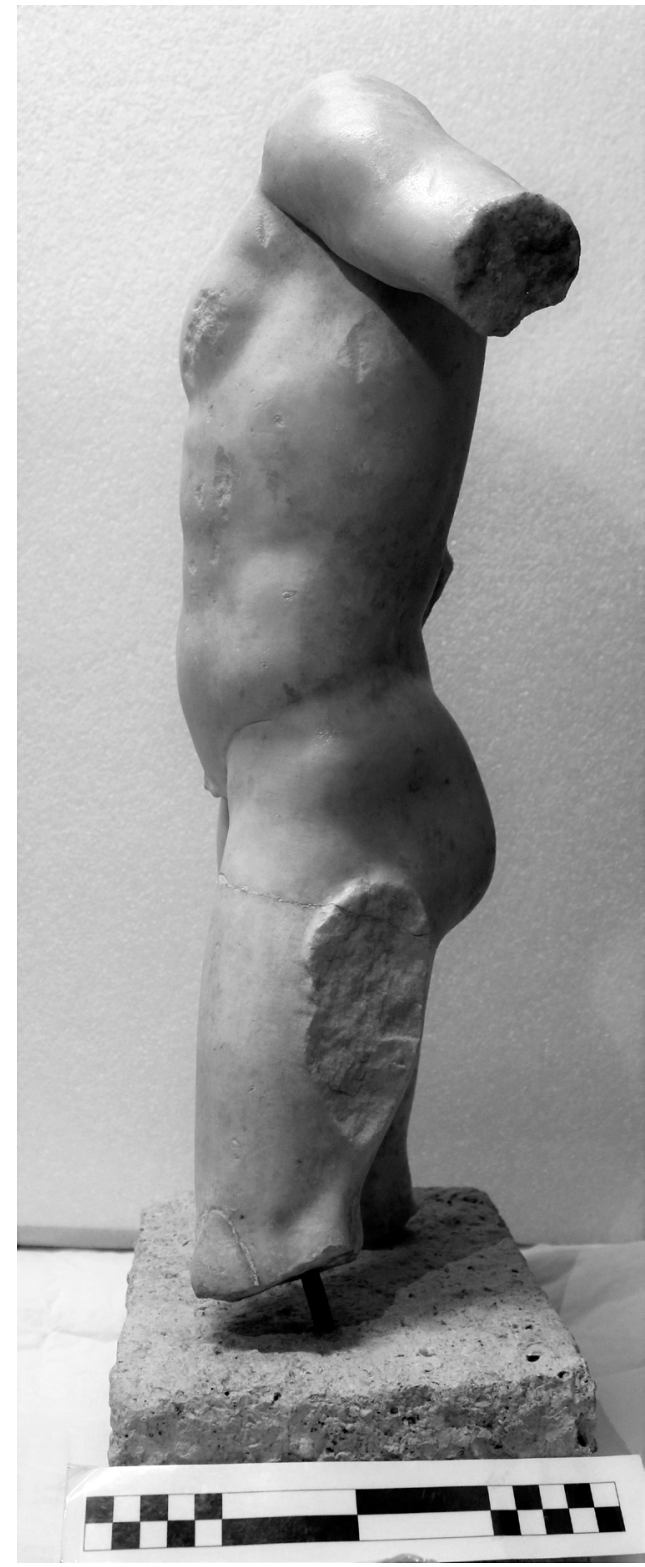

Figura 2: Torso. Lateral izquierdo. Foto: Cortesía Prof. J. M. Noguera Celdrán

Se trata de un torso masculino desnudo ${ }^{2}$ de mármol blanco de grano fino, acéfalo (Fig. 1). Está falto de brazos, de los que conserva tan solo el arranque desde el hombro en el lado derecho y algo más en el izquierdo, con la circunstancia de que se advierte cómo éste queda retraído hacia atrás separándose claramente del cuerpo (Fig. 2). Teniendo en cuenta la posición de las piernas, la diestra sosteniendo el peso del cuerpo y la izquierda retraída avanzando el muslo hacia adelante, se hace evidente una inestabilidad corporal, que haría necesaria la presencia de un apoyo sobre otra figura o tal vez,

2. N. ${ }^{\circ}$ de Inventario CE946. Alto conservado en la actualidad: 50 centímetros. Tenía concreciones que fueron eliminadas tras su restauración, así como también fueron restauradas las roturas que se advierten en ambas piernas. 


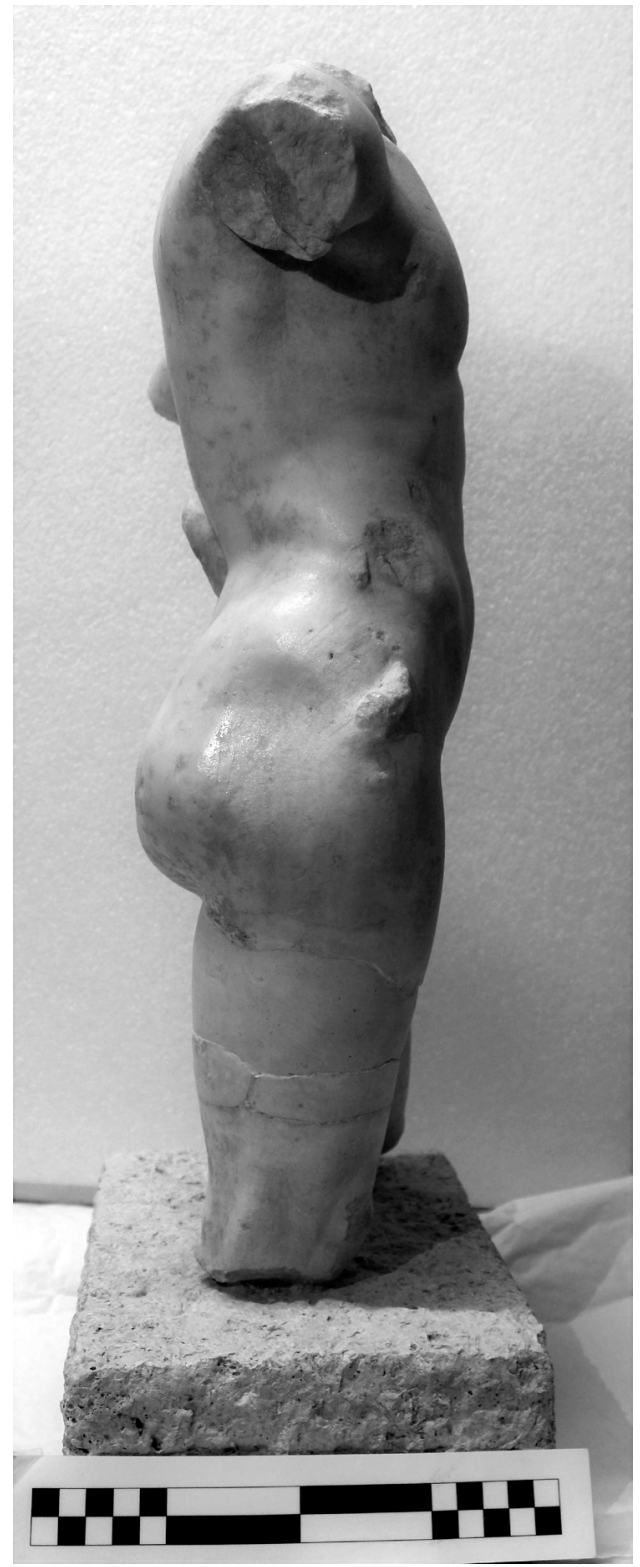

Figura 3: Torso. Lateral derecho. Foto: Cortesía Prof. J. M. Noguera Celdrán

más probable, en un tronco de árbol o pilar, refrendado por la rotura vertical que se advierte en el muslo del mismo lado ${ }^{3}$ (Fig. 2). Por el contrario, el brazo derecho -a tenor de la fractura que se aprecia- caería a lo largo del cuerpo, conservándose restos, tal vez de la mano o de un objeto no identificado, sobre la cadera (Fig. 3). A todo lo anterior ha de añadirse otro aditamento en la espalda, también de problemática filiación (Fig. 4). Con respecto al conjunto de la escultura ha de destacarse un correcto modelado que estudia la anatomía con bastante

3. Aragoneses (1957: 108) añade otras medidas del cuerpo, y en nota 3, especifica que la huella en el muslo es de forma ovalada de once por seis centímetros. Se advierten también erosiones en el pectoral izquierdo y en la zona lateral del abdomen.

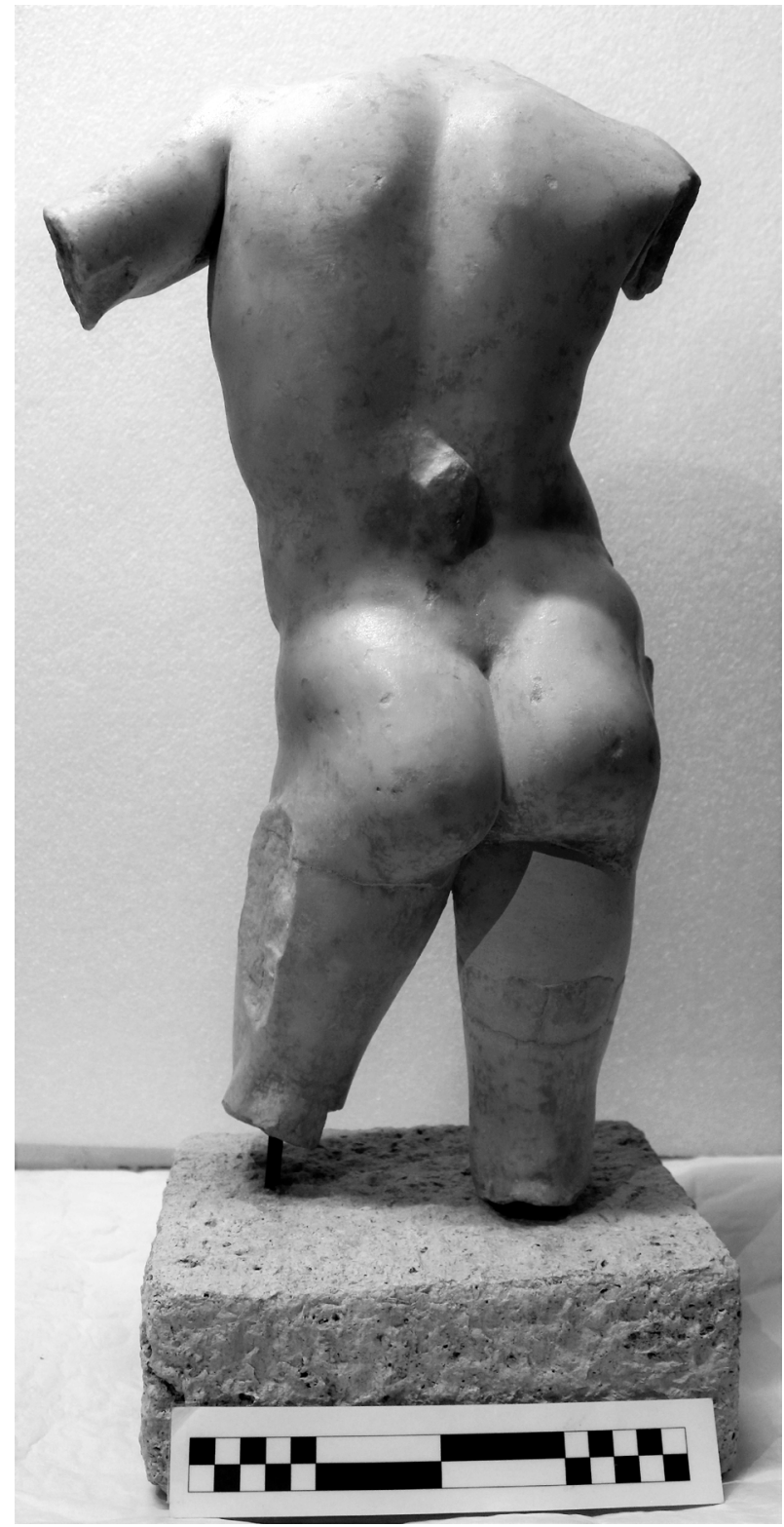

Figura 4: Torso. Visión trasera. Foto: Cortesía Prof. J. M. Noguera Celdrán

precisión, marcando el consabido quiasmo y los músculos pectorales, los abdominales y los de la espalda, notables en su ejecución para el tamaño de la escultura.

El primer editor científico de la escultura, Manuel Jorge Aragoneses, en el año 1957, basándose en estos elementos pensó en una figura de Apolo tomando como modelos, tan solo, los dibujos de Reinach. (Aragoneses, 1957: 108, notas 3-6; Reinach, 1904 (1965 ed. anast.): I, 239, n. ${ }^{\circ} 4 ; 241$, n. ${ }^{\circ} 5 ; 242$, n. $^{\circ} 1-3 ; 252$, n. ${ }^{\circ} 6$; V, 1924 (1969, ed. anast.): 474, n. ${ }^{\circ}$ 5; VI; 1930 (1969, ed. anast.): 183, n. $\left.^{\circ} 8\right)$. Faltaba, sin embargo, una tipología concreta y una posible reconstrucción hipotética de la escultura.

Para resolver la primera cuestión debemos fijarnos que, en nuestra escultura, se advierte claramente una estructura corporal que recuerda modelos de Policleto, muy en la línea del Diadúmeno o del Hércules del 
Museo Nazionale Romano y de otras figuras de atletas del mismo museo, entre otras muchas piezas que se podrían aducir ${ }^{4}$.

Pero es indudable que el ritmo de esta escultura bebe claramente en creaciones praxitélicas o de su escuela. A este respecto se ha efectuado un minucioso análisis, confrontando la pieza toledana con los repertorios modernos, llevándonos a la conclusión de que habría de ser, siguiendo la opinión de su primer editor, una figura de Apolo. Dentro de la iconografía del dios, el prototipo escultórico más acorde con la pieza toledana es, sin duda alguna, el Apolo Likeios, creación del propio Praxíteles en sus últimos años o quizás de sus seguidores, de la que derivarán innumerables copias y variaciones durante el helenismo y la época romana ${ }^{5}$. Tomando como punto de partida este arquetipo escultórico, parece oportuno mostrar en rápida sucesión una de las posibles transformaciones del modelo, que desembocarán con el paso del tiempo en el tipo estatuario que nos ocupa. En relación con esta creación praxitélica recordemos algunos excelentes ejemplares, entre los cuales podemos mencionar los del Louvre, (Rizzo, 1932: 79 ss., láms. 109 ss.; Lippold, 1950: 84,1; Bieber, 1955: 18, fig. 17; De Franciscis, 1958: 468-470; Palagia, 1984: 193-194, 39f (dos ejemplares $n^{\circ} \mathrm{s}$ inv. 910 y 928); Simon y Bauchhenss, 1984: n. ${ }^{\circ}$ 54.), Atenas, (Bieber, 1955: 18, figs. 20-21; Becatti, 1965: 429, fig. 470; Palagia, 1984: 194, n. ${ }^{\circ}$ 39r; Simon y Bauchhenss, 1984: 379-380, n. ${ }^{\circ}$ 54k), Venecia (Traversari, 1973: 92-93, n. ${ }^{\circ}$ 36; Palagia, 1984: 194, n. ${ }^{\circ} 39$; : Simon y Bauchhenss, 1984: 380, n. ${ }^{\circ}$ 55) y Florencia ${ }^{6}$, fechados en distintos momentos. En todos ellos observamos la gran semejanza en el movimiento de sus miembros con la escultura toledana a excepción de la posición del brazo derecho.

A partir de segunda mitad del siglo II a. C., según la opinión de eminentes especialistas, se adaptará el tipo del Lykeios para crear la figura del Apolo Citaredo (De Petra, 1872; Wernicke, 1895: 100-111; Bieber, 1955:

4. Paribeni, 1953: 37, n. ${ }^{\circ} 53$, n. $^{\circ}$ inv.106184; Steuben, 1969: 159160, n. ${ }^{\circ} 2251$; Candilio, 1979: 214-215, n. ${ }^{\circ} 132$ con abundante bibliografía anterior. Particularmente interesante es el Efebo de Dresde (Lippold, 1950: 166) que ofrece desde el punto de vista anatómico concordancias con la pieza toledana. Vid. infra nota 10 .

5. Abundantísima la bibliografía sobre esta famosa escultura. Klein (1898: 158-181) con clasificación tipológica y elenco de copias y variantes, con crítica de las mismas por Deubner, 1934: 26-45 y 62-65; Rizzo, 1932: 79-85; Lippold, 1950: 238-239, lám. 84,1; Picard, 1954: 327-351, nota n. ${ }^{\circ} 2,327$, nota n. ${ }^{\circ}$ 1, 334; Becatti, 1965: 429; Palagia, 1984: 193, n. ${ }^{\circ}$ 39; Simon y Bauchhenss, 1984: 179, n. ${ }^{\circ}$ 54, con numerosas réplicas. Sobre Praxíteles en general, más recientemente, en Pasquier y Martinez, 2007.

6. Llamado «Apollino»: Deubner, 1934: 40-45, 75; Lippold, 1950: 329; Bieber, 1955: 18; Mansuelli, 1958: 74-76, n. ${ }^{\circ} 46$ con amplio estudio y bibliografía; De Francicis, 1958: 470, fig. 638; Palagia, 1984: n. ${ }^{\circ}$ 39aa; Simon y Bauchhenss, 1984: 380, n. $^{\circ} 55$.
160; De Franciscis, 1958: 471; Steuben, 1966: 191; Palagia, 1984: 193), primero en su acepción como figura semidesnuda, ataviada tan solo con la clámide cubriendo las piernas y apoyando el brazo izquierdo sobre la cítara. Valgan como ejemplos la figura conservada en el Museo Británico ${ }^{7}$, procedente de Cirene y la del Museo Capitolino (Stuart Jones, 1912: 279, n. ${ }^{\circ}$ 7, lám. 67; Bieber, 1955: 160, fig. 680; Steuben, 1966: 190-191, n. ${ }^{\circ} 1383$, n. $^{\circ}$ inv. 628; Palagia, 1984: 212, n. ${ }^{\circ}$ 222; Simon y Bauchhenss, 1984: 384, n. ${ }^{\circ} 61 \mathrm{k}$ ) hallada en el Esquilino, entre la multitud de copias existentes. En ellas existe una clara reelaboración en las que se advierte un marcado barroquismo ornamental. A la figura del dios se le adosan, además del tocón arbóreo, los atributos que le son propios, esto es, la cítara, el trípode délfico, el grifo y la serpiente Pitón, a las que se añadirá en otras esculturas, el cisne. De esta forma las figuras ganan en riqueza compositiva y belleza gracias a la calidad del trabajo escultórico.

Poco después ya aparecerá el dios desnudo, adaptándose a los gustos del último helenismo. El dios se nos muestra de pie, con la cabeza erguida, el brazo izquierdo separado del cuerpo portando la cítara que apoya en un pilar, al que se junta la pierna, para mayor estabilidad, mientras que el brazo derecho, sigue manteniendo la posición canónica del brazo levantado sobre la cabeza. Este es el tipo del Apolo de Dresde (Palagia, 1984: 194, n. ${ }^{\circ}$ 39n) y el de Roma, también en el Museo Capitolino (Stuart Jones, 1912: 346, n. ${ }^{\circ}$ 7, lám. 86; Rizzo, 1932: 81, lám. 119 y 126; Lippold, 1950: 238, nota 7; Picard, 1954: 340, fig. 145; Steuben, 1966: 232-233, n. ${ }^{\circ}$ 146; Palagia, 1984: 209, n. ${ }^{\circ}$ 196; Simon y Bauchhenss, 1984: 179, n. ${ }^{\circ}$ 54h).

De momentos posteriores, singularmente de finales del helenismo y la primera época imperial romana, siguiendo estos mismos modelos existen otras esculturas que han sufrido una simplificación de elementos ornamentales huyendo de recargar la figura y se ha producido el cambio en la postura del brazo derecho que se dispone ahora recto a lo largo del cuerpo, apoyando

7. Imagen identificada por la mayoría de los especialistas con la estatua esculpida por el ateniense Timarquides para el templo de Apolo in Campo (luego Sosiano) por encargo de M. Emilio Lepido en el añ0 179 a. C., según noticia transmitida por Plinio, N.H. 36,35; Moreno, 1966: 856-857; Deubner, 1934: 63 ss., n. ${ }^{\circ} 30$; Becatti, 1935: 121ss. figs, 2 y 9; Lippold, 1950: 329 , nota 7, también con amplia bibliografía; Bieber, 1955: 160, figs. 678-679; Huskinson, 1975: 6-7, n. ${ }^{\circ}$ 12, lám. 5, Palagia, 1984: 211-212. n. ${ }^{\circ} 222$; Simon y Bauchhenss, 1984: 383, n. $^{\circ}$ 61, con bibliografía y lista de copias; Moreno, 1994: 522-523, figs. 642-646. Debe recordarse la estatua del Museo del Prado en la que se representa este tipo escultórico con variantes: Blanco, 1957: 92, n. ${ }^{\circ}$ 155, lám. 74; Schröder, 2004: 246-248, cat. n. ${ }^{\circ} 147$. Más cercana a la del Museo Británico, citada, y a la del Museo Capitolino, de la nota que sigue, es una estatua fragmentaria que conserva la parte inferior, procedente de Carteia: Rodríguez Oliva, 1994: 154-157, figs. de las pp. 153 y 156 . 


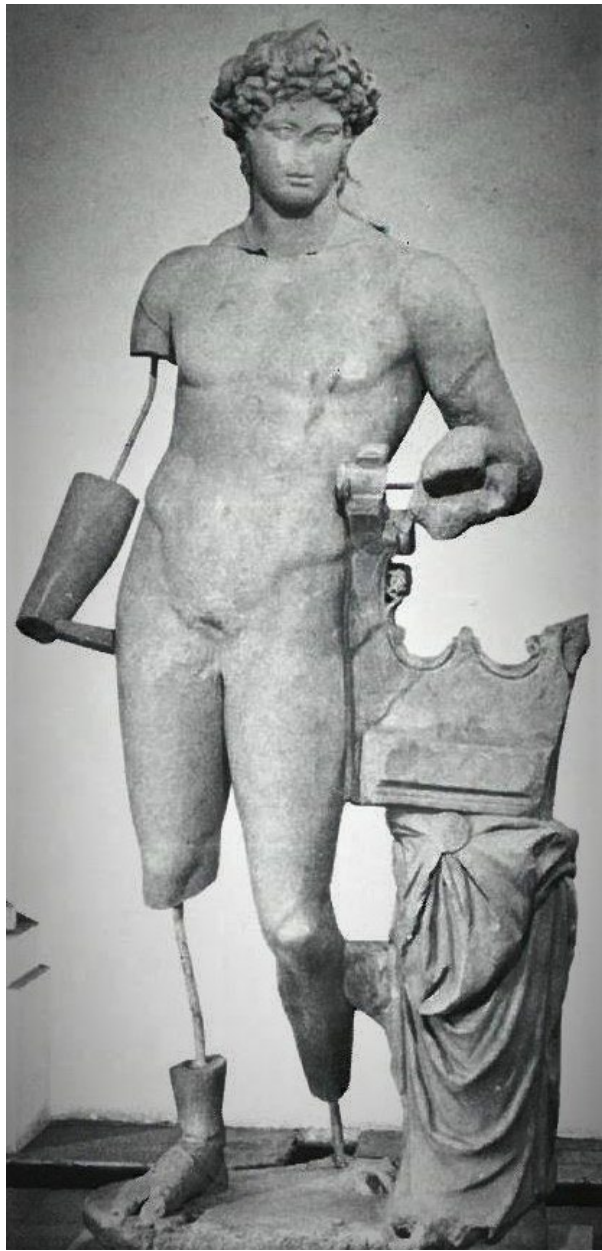

Figura 5: Apolo Citaredo. Museo de Nicosia (Chipre) Según LIMC, II, 2, 202, $\mathrm{n}^{\circ} 221$

unas veces la mano sobre la cadera y otras llevando el plectro.

Conviviendo con las estatuas de grandes dimensiones, estas esculturas por lo general de menor tamaño, surgen para ser adaptadas a jardines y peristilos de las villae repartidas por todo el imperio romano, especialmente durante el siglo I d. C. y la primera mitad del II d. C. Es el caso de una escultura que se guarda en el Museo de Nicosia, procedente de Salamina (Fig. 5) (Palagia, 1984: 211, n. ${ }^{\circ} 221$; Simon y Bauchhenss, 1984: 386, n. ${ }^{\circ}$ 67) que se considera derivación del Apolo Likeios, la cual es cabeza de serie de un nutrido grupo de esculturas similares, caso de los ejemplares de Estambul (Fig. 6) (Mendel, 1914: 79-80, n. ${ }^{\circ}$ 317; Simon y Bauchhenss, 1984: 386, n. ${ }^{\circ}$ 67d) y de Cleveland, Ohio, Museum of Art (Fig. 7) (Palagia, 1984: 211, n. ${ }^{\circ}$ 220; Simon y Bauchhenss, 1984: 387, n. $\left.{ }^{\circ} 68\right)$ a las que pueden añadir otras con diversas variantes en diferentes museos.

En mi opinión, habría que adscribir la pieza toledana a estos últimos tipos escultóricos por su evidente semejanza, modelos que habrán de servir para una futura reconstrucción ideal de nuestra escultura, que

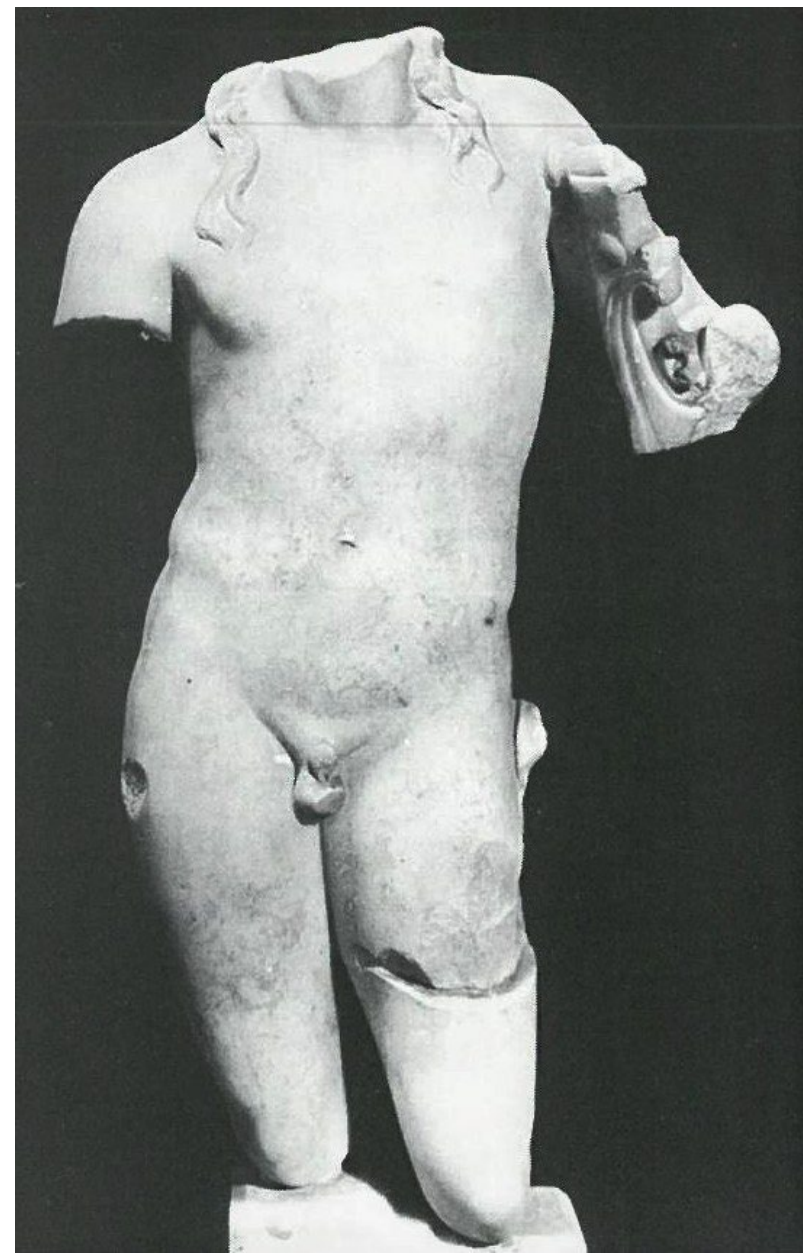

Figura 6: Apolo Citaredo. Museo de Estambul. Según LIMC, II, 2, 306, $\mathrm{n}^{\mathrm{o}} 67 \mathrm{~d}$

fechamos en la primera mitad del siglo II d. C. basándonos en los ejemplares aducidos, los cuales tienen la misma data. Como mejor paralelo para el torso toledano en su estado actual, al menos que sepamos, ya lo apuntó su primer editor sobre el dibujo de Reinach (Rep. St., VI, 1930, 183, n. ${ }^{\circ}$ 8), que reproduce la escultura de un torso masculino de mármol de Paros y de dimensiones similares al ejemplar toledano, que perteneció a la antigua colección de Sir Alfred Mond, Primer Barón Melchet de Landford (Figs. 8 y 9), que la conservaba en su colección de Melchet Court, en Hampshire (Strong, 1928: 14, lám. XV, n. ${ }^{\circ} 7$ ).

Además de todo lo expuesto habría que considerar la hipótesis de que esta escultura toledana formase parte de un grupo formado por Dionysos y un sátiro ${ }^{8}$. Porque no cabe duda que la pose del Apolo Likeios fue aprovechada por los copistas para trasponer el tipo apolíneo a esta divinidad, la cual no solo se apoya en pilares o tocones de árboles, sino en el cuerpo de un sátiro o de

8. Idea que me fue sugerida por el profesor Armando Cristilli, a quien agradezco cordialmente la observación. 


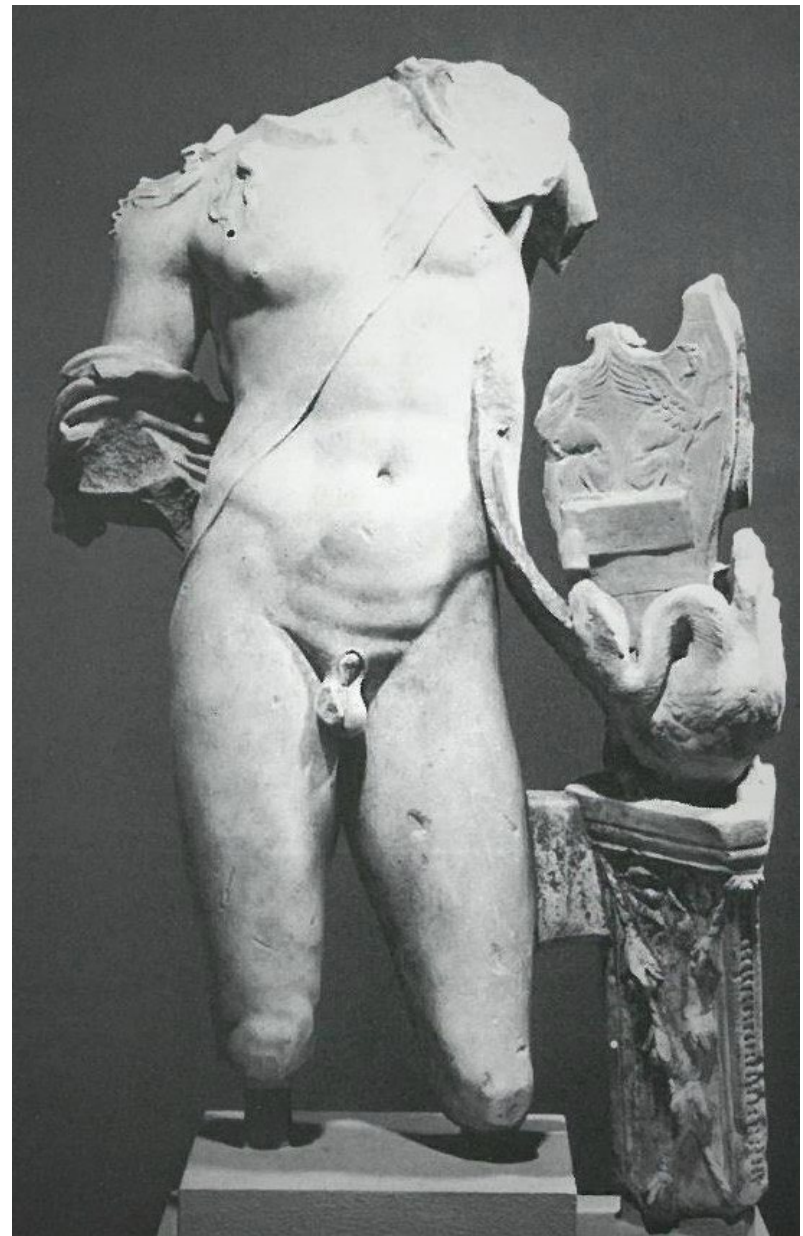

Figura 7: Apolo Citaredo. Museo de Cleveland (Ohio). Según LIMC, II, 306, $\mathrm{n}^{\circ} 68$

Sileno u otros miembros de su thiasos ${ }^{9}$. Estos suelen abrazar al dios por la cintura cogiéndolo por el costado izquierdo. Esto podría explicar la presencia del aditamento que se observa en la zona lumbar de la espalda de nuestra figura como elemento de sujeción del brazo, pero no poseemos la certeza de otros elementos que puedan confirmar plenamente esta idea ${ }^{10}$. Por otra parte, los ejemplares de Nicosia o de Cleveland, antes mencionados, que consideramos como paralelos cercanos de la estatua toledana, son coetáneos con otras esculturas

9. Véase al respecto, por poner dos ejemplos significativos, el grupo del Museo Nazionale Romano, perteneciente a la colección Ludovisi (Steuben, 1969: 286-287, n. ${ }^{\circ} 2358$; Palma, 1983: 101, n. ${ }^{\circ} 42$ ), ambos con bibliografía previa, y el de Venecia (Traversari, 1982: 43-46). Para la tipología general de Dionysos representado solo o en grupo, cfr. Gasparri (1986: 540-566).

10. Fundamentalmente si se tiene en cuenta la estructura corporal, como pone de manifiesto Schröder (2004: 356-361, n. ${ }^{\circ}$ 179) comparando la figura de un joven Dionysos, conservado en el Museo del Prado, de anatomía suave y rasgos musculares atenuados, con el Efebo del Albertinum de Dresde, el cual posee una musculatura bien definida similar al torso toledano.

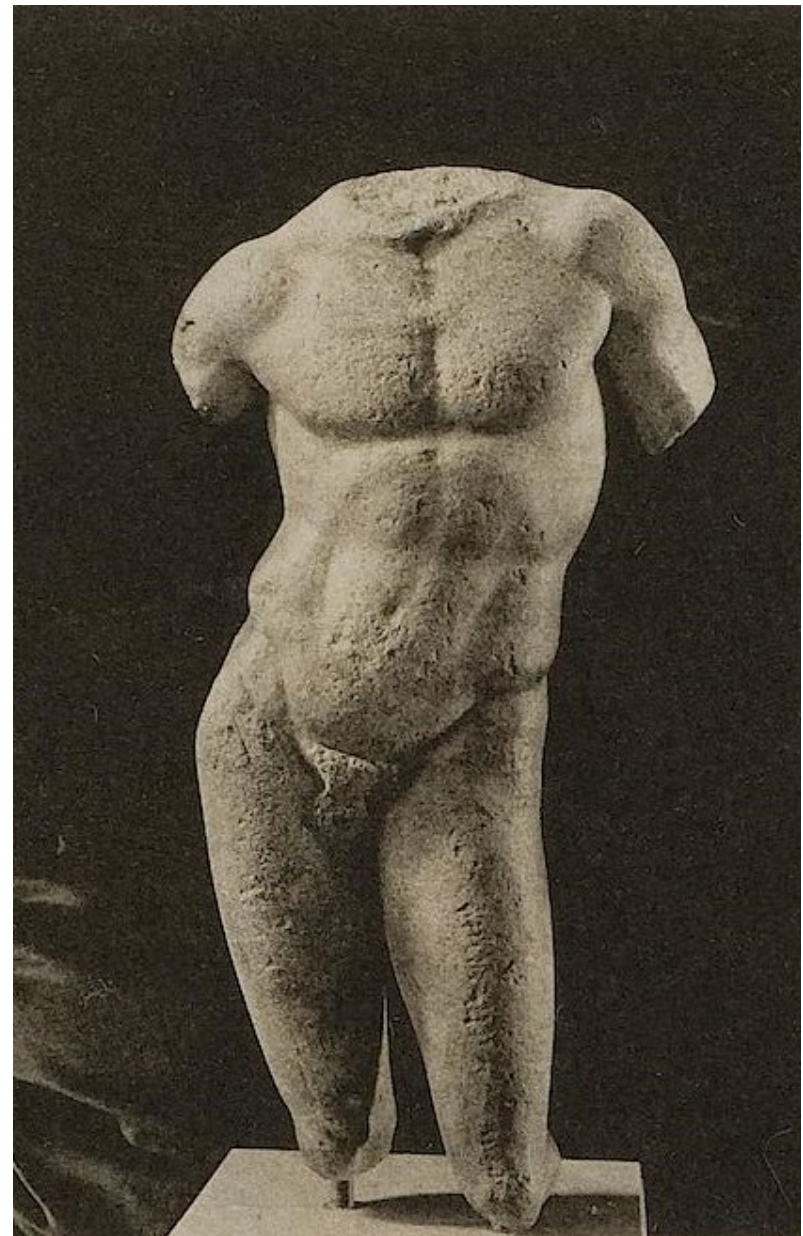

Figura 8: Torso. Visión frontal. Antigua Colección Melchet. Según Strong, 1928: lám. XV, 7

que son recuerdos lejanos de los prototipos originales a causa de una marcada estilización de aquellos antiguos modelos. Son, generalmente, figuras individuales que representan a otros personajes mitológicos como Narciso o Jacinto ${ }^{11} \mathrm{o}$, simplemente, a jóvenes atletas ${ }^{12}$, los cuales se podrían presuponer como análogos a la escultura en estudio. El estado fragmentario de la escultura solo permite moverse en el campo incierto de las hipótesis, dejando abierta la interpretación de esta escultura para futuros trabajos.

Como conclusión a las líneas precedentes hay que añadir que la pieza escultórica estudiada, desgraciadamente de una procedencia desconocida, debió formar parte de la decoración del peristilo de una villa, posiblemente con otras figuras mitológicas. La identificación de la escultura con un Apolo, que hemos sostenido en

11. Es el caso de una escultura procedente de una villa, también de la provincia de Toledo, publicada recientemente por Rodríguez Oliva (2018: 351-364, figs.1, 2b, 3a). Ejemplar completo en el Museo del Prado (Blanco, 1957: 84, n. ${ }^{\circ}$ 124E, lám. 74; Schröder, 2004: 74-79, n. ${ }^{\circ}$ 105).

12. Como sucede en el llamado «Joven orador» en el Museo del Prado: Blanco, 1957: 40-41, n. ${ }^{\circ}$ 39E, lám. 21; Schröder, 2004: 248-252, n. ${ }^{\circ} 148$. 


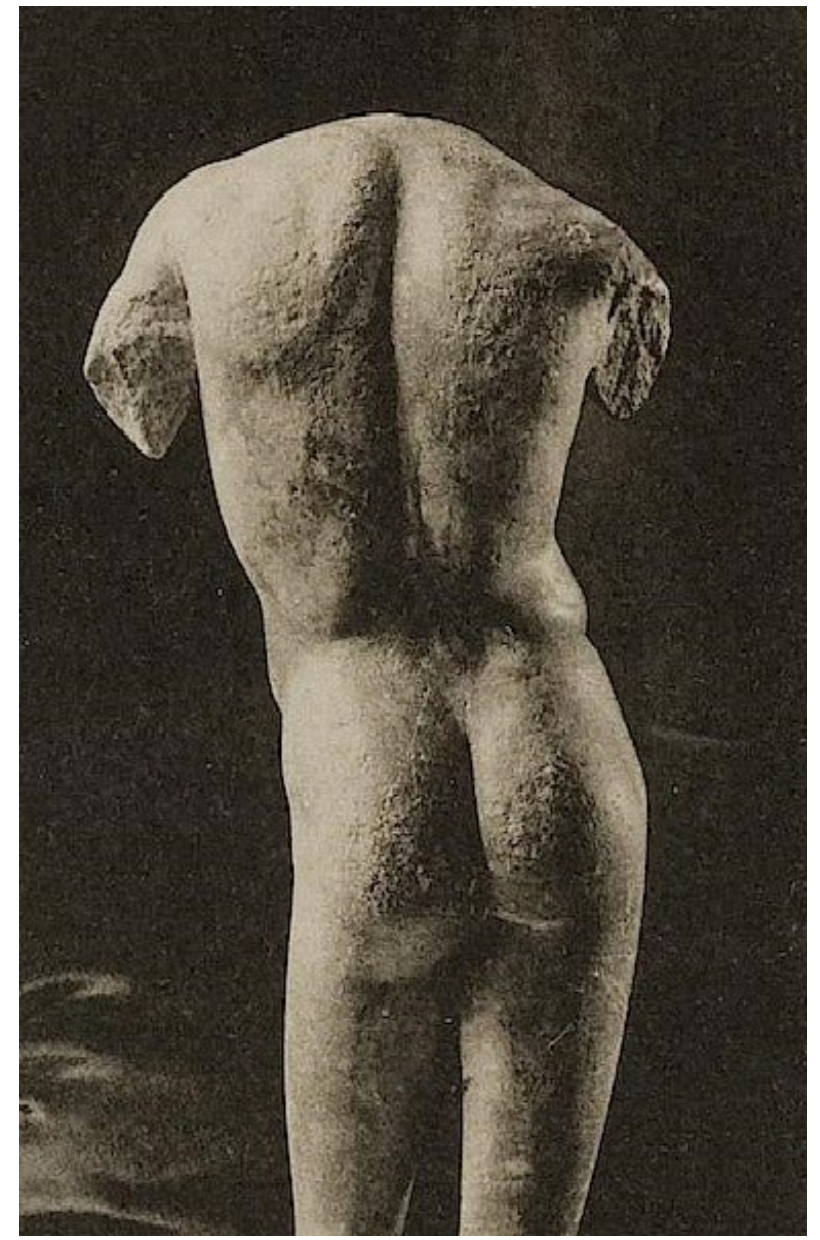

Figura 9: Torso. Visión trasera. Antigua Colección Melchet. Según Strong, 1928: lám. XV, 7

este escrito, es la que nos parece más plausible al estar en consonancia en muchos detalles con la iconografía del dios y con la cronología establecida para los paralelos más cercanos. No está en contradicción con la conjetura de que pudiera ser Dionysos solo o, mejor, formando un grupo, sin descartar la suposición de otras esculturas con una tipología de similares características. Queda, pues, reservada para futuros trabajos la tarea de profundización sobre la identidad de esta interesante escultura.

\section{REFERENCIAS}

Aragoneses, M. J. (1957). El torso varonil romano en el Museo Arqueológico de Toledo. Archivo Español de Arqueología, 29-30, 106-108.

Aragoneses, M. J. (1958). Museo Arqueológico de Toledo. Madrid: Dirección General de Bellas Artes.

Baena del Alcázar, L. (2016). Sobre un torso masculino en el Museo de Santa Cruz (Toledo). En Preactas de la VIII Reunión de Escultura Romana en Hispania (Córdoba Baena, 5 al 8 de octubre) (pp. 79-80). Córdoba: Editorial Universidad de Córdoba.
Becatti, G. (1935). Timarchides e l'Apollo «qui tenet citharam». Bullettino della Commissione Archeologica Comunale di Roma, LXIII, 111-131.

Becatti, G. (1965). Prassitele. En Enciclopedia dell'Arte Antica, vol. VI (pp. 423-431). Roma: Ed. Treccani.

Bieber, M. (1955). The Sculpture of the Hellenistic Age. New York: Hacker Art Books.

Blanco Freijeiro, A. (1957). Catálogo de la Escultura. Museo del Prado. Madrid: Patronato Nacional de Museos.

Candilio, D. (1979). En Museo Nazionale Romano. Le Sculture, I (pp. 214-215, n. ${ }^{\circ} 132$ ). Roma: De Luca.

Deubner, O. (1934). Hellenistische Apollogestalten. Athen: Buchdruckerei Hestia.

Gasparri, C. (1986). Dionysos. En Lexicon Iconographicum Mythologiae Classicae, III (pp. 240-514). Zürich - München: Artemis Verlag.

Gasparri, C. (1986). Dionysos - Bacchus. En Lexicon Iconographicum Mythologiae Classicae, III (pp. 540-566). Zürich - München: Artemis Verlag.

Franciscis, A. de (1958). Apollo. En Enciclopedia dell'Arte Antica, I (pp. 463-473). Roma: Ed. Treccani.

Huskinson, J. (1975). Roman Sculpture from Cyrenaica in the British Museum. C.S.I.R. Great Britain, II, 1. London: British Museum Publications Ltd.

Klein, W. (1898). Praxiteles. Leipzig: Verlag von Veit \& Comp.

Lambrinudakis, W. y Palagia, O. (1984) Apollon. En Lexicon Iconographicum Mythologiae Classicae, II (pp. 183-327). Zürich - München: Artemis Verlag.

Lippold, G. (1950). Die griechische Plastik, Handbuch der Archáologie, III, 1. München: Beck.

Mansuelli, G. A. (1958). Galleria degli Uffizi. Le Sculture, I. Roma: Istituto Poligrafico dello Stato, Libreria dello Stato.

Mendel, G. (1914). Catalogue des Sculptures grecques, romaines et byzantines, vol. II. Constantinople: Musée Imperiaux Ottomans.

Moreno, P. (1966). Timarchides. En Enciclopedia dell'Arte Antica, VII (856-857). Roma: Ed. Treccani.

Moreno, P. (1994). Scultura ellenistica. Roma: Istituto Poligrafico e Zecca dello Stato.

Palma, B. (1983). En Museo Nazionale Romano. Le Sculture, I, 5 (p. 101). Roma: De Luca.

Pasquier, A. y Martinez, J. L. (Eds.). (2007). Praxitèle. Paris: Musée du Louvre Editions.

Paribeni, E. (1953). Museo Nazionale Romano. Sculture Greche del V secolo. Roma: De Luca.

Petra, G. de (1872). Sullo sviluppo del tipo di Apollo. Napoli: Tipografia editr. degli Accattonoelli. 
Picard, Ch. (1954). Manuel d'Archéologie Grecque. La Sculpture, IV, 2. Paris: Editions A. et J. Picard.

Reinach, S. (1904, 1969 ss. ed. anastática). Répertoire de la Statuaire Grecque et Romaine, I-VI. Paris: Ernest Leroux, Éditeur.

Rizzo, G. E. (1933). Prassitele. Roma - Milano: Treves Treccani - Tumminelli.

Rodríguez Oliva, P. (1994). Sobre el culto de Apolo en la Bética. En Latinitas biblica et christiana: Studia philologica varia in honorem Olegario García de la Fuente (pp. 144158). Madrid: Universidad Europea de Madrid.

Rodríguez Oliva, P. (2018). Una nueva copia romana del «Narciso»/«Jacinto» de Policleto encontrada en una villa de la provincia de Toledo. En Escultura romana en Hispania VIII (pp. 361-364). Córdoba: UCO Press, Editorial Universidad de Córdoba.

San Román, F. de B. (1941). Museo Arqueológico de Toledo. Memoria. Memoria de los Museos Arqueologicos Provinciales, II, 153.

Schröder, S. F. (2004). Catálogo de la escultura clásica. Museo Nacional del Prado, vol. II. Madrid: Museo del Prado.

Simon, E. y Bauchhenss, G. (1984). Apollon - Apollo. En Lexicon Iconographicum Mythologiae Classicae, II (pp. 363446). Zürich - München: Artemis Verlag.
Steuben, H. von (1966). En W. Helbig. Führer durch die öffentlichen Sammlungen klassischer Altertümer in Rom, II (pp. 190-191, n. ${ }^{\circ}$ 1383). Tübingen: E. Wasmuth.

Steuben, H. von (1969). En W. Helbig. Führer durch die öffentlichen Sammlungen klassischer Altertümer in Rom, III (pp. 286-287, n. ${ }^{\circ}$ 2358). Tübingen: E. Wasmuth.

Strong, E. (1928). Catalogue of the Greek \& Roman Antiques in the possession of the Right Honourable Lord Melchett, at Melchet Court and Lowndes Square. Oxford - London: Oxford University Press.

Stuart Jones, H. (1912). A Catalogue of Ancient Sculpture preserved in the Municipal Collection of Rome. The Museo Capitolino. Oxford: Clarendon Press.

Traversari, G. (1973). Sculture del V.-IV. Secolo a.C. del Museo Archeologigo di Venezia. Roma: Alfieri.

Traversari, G. (1982). Il gruppo de Dioniso appogiato ad un satiro nel Museo Archeologico di Venezia, Rivista di Archeologia, VI, 43-46.

Wernicke, K. (1895). Apollon. En A. F. von Pauly y G. Wissowa. Realencyclopädie der klassischen Altertumwissenschaft, III, 1 (cols. 33-41 y 100-111). Stuttgart: Metzler-Verlag. 